\title{
Supraspinatus muscle location changes after arthroscopic rotator cuff repair: a potential source of preoperatively predicting tear patterns
}

This article was published in the following Dove Medical Press journal: Open Access Journal of Sports Medicine

\section{Yohei Ono' \\ Takeshi Makihara ${ }^{2}$ \\ Satoshi Yamashita ${ }^{3}$ \\ Kelvin KW Tam ${ }^{4}$ \\ Nobuaki Kawai' \\ lan KY Lo ${ }^{5}$ \\ Akihiko Kimura'}

'Department of Orthopaedic Surgery, East Hokkaido Hospital, Kushiro, Hokkaido, Japan; ${ }^{2}$ Department of Orthopaedic Surgery, Faculty of Medicine, University of Tsukuba, Tsukuba, Ibaraki, Japan; ${ }^{3}$ Department of Orthopaedic Surgery, Nagoya University Graduate School of Medicine, Showa-ku, Nagoya, Japan; ${ }^{4}$ Asia Medical Specialists, Hong Kong, China; ${ }^{5}$ Department of Surgery, Section of Orthopaedic Surgery, McCaig Institute for Bone and Joint Health, University of Calgary, NW, Calgary, AB, Canada
Correspondence: Yohei Ono Department of Orthopaedic Surgery, East Hokkaido Hospital, 7-19 Wakatake-cho, Kushiro, Hokkaido 085-0036, Japan

$\mathrm{Tel}+8 \mathrm{I} I 54233425$

Email y.8.show@gmail.com
Purpose: When repairing retracted rotator cuff tears, the tear pattern (eg, crescent-shaped, L-shaped) is best determined intraoperatively by evaluating the mobility of the tendon in multiple directions. The purpose of our study was to evaluate the location of the supraspinatus (SSP) muscle belly on magnetic resonance imaging (MRI) in patients undergoing arthroscopic repair of retracted rotator cuff tears. We hypothesized that the location of the rotator cuff muscle would move after tendon repair, and that the perioperative change in muscle position would correlate with the tear pattern.

Methods: A series of primary arthroscopic repairs for rotator cuff tears with $>3 \mathrm{~cm}$ of medial retraction from 2015 to 2016 was reviewed. MRIs were performed preoperatively and within 10 days postoperatively. The SSP muscle was assessed on sagittal MRI and evaluated for the "occupation ratio", "tangent sign", and the "location index" proposed in this study. Pre and postoperative MRIs were compared, and correlated with intraoperatively determined tear patterns. Fifty shoulders without rotator cuff pathology were also assessed for the "location index" as control. Results: Fifty-nine shoulders (mean age 65.0 years) were included, among which five reverse L-shaped tears were identified. The occupation ratio and tangent sign improved postoperatively. Preoperatively, in the majority of tears, the SSP muscle was located more posteriorly in the SSP fossa, compared to the control group, and shifted anteriorly after repair. However, in reverse L-shaped tears the SSP muscle was located more centrally in the fossa, and shifted posteriorly following repair. Conclusion: Rotator cuff tearing and arthroscopic rotator cuff repair change the location of the SSP muscle. Although repair usually results in shifting of the muscle belly from posterior to anterior, reverse L-shaped tears demonstrated an opposite pattern. The location of the SSP muscle belly may be useful in predicting tear patterns of retracted rotator cuff tears.

Level of evidence: Level IV (case series).

Keywords: shoulder, rotator cuff, muscle belly, muscle location, MRI, muscle atrophy, tendon retraction, location index

\section{Introduction}

Surgical repair of large to massive retracted rotator cuff tears can be technically challenging and thus understanding its characteristics is essential. ${ }^{1}$ Preoperatively, the repairability of a rotator cuff tear may be assessed by the tear size, ${ }^{2}$ retraction, ${ }^{3}$ fatty infiltration, ${ }^{4,5}$ and atrophy ${ }^{6,7}$ of cuff muscles on magnetic resonance imaging (MRI). However, the tear pattern (eg, crescent-shaped, L-shaped, reverse L-shaped, U-shaped tear), ${ }^{1,8-11}$ is best evaluated intraoperatively where the inherent medial to lateral and anterior to posterior mobility of the torn tendon can be directly evaluated. 
Degenerative changes in the rotator cuff muscles (ie, fatty infiltration, atrophy) have been associated with poor tendon healing and inferior clinical outcomes following surgical repair. ${ }^{12,13}$ While several studies have demonstrated the importance of preoperative MRI assessment, the changes occurring to the rotator cuff muscles immediately postoperatively and in particular the position of the muscle belly have rarely been investigated. Indeed, immediate postoperative MRI has demonstrated some improvement in the muscular atrophy and fatty infiltration of the muscle belly of supraspinatus (SSP) and infraspinatus (ISP) following repair. ${ }^{14}$ These findings have recently been shown to continue to improve even up to 2 years following surgery. ${ }^{15}$ This suggests that reducing the tendon and restoring the normal length tendon relationship of the rotator cuff may be important. However, the effect of various tear patterns, and therefore the direction of tendon reduction on the position of the SSP muscle belly, has not to our knowledge been previously investigated.

Given that immediate sagittal MRI views demonstrate recovery in the appearance of the rotator cuff muscle belly after surgical repair, ${ }^{14}$ they may also demonstrate variable patterns of muscle recovery depending on the tear pattern. Furthermore, recognizing how the location of the muscle belly changes with tearing and following repair may help predict the tear pattern. This may assist the arthroscopist when surgically repairing the tendon.

Therefore, the purpose of our study was to evaluate the perioperative changes of the rotator cuff muscle belly on MRI, with a particular focus on the location of SSP muscle, in patients undergoing arthroscopic rotator cuff repair. We hypothesized that the location of the SSP muscle belly would vary before repair and would be restored toward the center of the SSP fossa after repair. Furthermore, different tear patterns and the subsequent tear reduction would correlate to the muscle belly location.

\section{Methods}

\section{Study design and patient enrollment}

This is a retrospective study involving a review of the medical records of patients who underwent primary arthroscopic rotator cuff repair from July 2015 to July 2016. Patients were operated on and followed postoperatively by the senior author, a shoulder surgeon with $>20$ years of experience. Study candidates were identified from a billing review and contacted by the research coordinator to determine their interest in participating in the study. This study was approved by the East Hokkaido Research Ethics Board (EH-REB\#16-02) and was conducted in accordance with the Declaration of Helsinki. Written consent forms were obtained from all patients.

\section{Inclusion and exclusion criteria}

Patients with a large to massive rotator cuff tear involving predominantly the SSP tendon, regardless of traumatic episodes, were included in the study. Tear size was determined by the retraction of the SSP tendon on preoperative coronal oblique MRI views using the Cofield classification. ${ }^{2}$ Patients were excluded from the study if there were other significant pathologies (eg, fracture, dislocation), cuff tear arthropathy (Hamada classification > grade 3), ${ }^{16}$ previous rotator cuff repair, or if insufficient information was available for the study.

\section{Surgical technique}

All patients were operated in a lateral decubitus position under general anesthesia. The arm was held with traction at $\sim 30^{\circ}$ of abduction. Following subacromial decompression, the mobility of the torn rotator cuff tendon was carefully assessed. As a principle, tendon repair was performed under minimum tension, determined by reducing the edges of the tendon to the bone bed with the use of a tendon grasper. Capsular release and the interval slides in continuity (ie, coracohumeral ligament release) ${ }^{17}$ were performed if necessary to achieve low tension repair. Double-row or triple-row repair (ie, suture bridge technique over a classic double-row construct) was performed if the tendon could cover more than half of the footprint width. Otherwise, single-row repair or partial repair was chosen if the tension appeared significant, in combination with footprint medialization if necessary.

\section{Imaging protocol}

The majority of preoperative MRIs were performed following a standardized protocol at the East Hokkaido Hospital. If the initial images were deemed inadequate (eg, poor quality, $>1$ year old) or there was any suspicion of tear progression, a new MRI was obtained. Seven patients (of 58) had MRIs from other facilities in the same region but were deemed sufficient for assessment and inclusion in the study. All patients routinely underwent MRI evaluation within 10 days (mean $4.2 \pm 1.4$ days) postoperatively utilizing the same standardized protocol to evaluate the changes of rotator cuff tendons and muscles after repair. Standard radiographs of the shoulder were also obtained preoperatively from all patients.

MRIs were performed utilizing a 1.5-T scanner (Optima MR 360 Advance; GE Healthcare Japan Corporation, Tokyo, 
Japan) equipped with a dedicated shoulder coil or a $0.4-\mathrm{T}$ scanner (APERTO Inspire; Hitachi Ltd, Tokyo, Japan). The affected arm was placed and kept at the side of the body in a neutral position. Of the multiple planes and sequences obtained, image assessment for the study was performed on T2-weighted coronal oblique and sagittal oblique images. The coronal oblique plane was oriented parallel to the scapular body whereas the sagittal oblique plane was oriented perpendicular to the scapula. The imaging settings were as follows: repetition time, 4,000-5,500 ms; echo time, $118 \mathrm{~ms}$; matrix, $288 \times 288$; slice thickness, $3 \mathrm{~mm}$; interslice gap, $1.2 \mathrm{~mm}$; field of view, $17 \times 17 \mathrm{~cm}$ for Optima MR 360 Advance; and repetition time, 3,350-5,000 ms; echo time, 126-162 ms; matrix, $192 \times 224$; slice thickness, $6 \mathrm{~mm}$; interslice gap, $1.0 \mathrm{~mm}$; field of view, $18 \times 18 \mathrm{~cm}$ for APERTO Inspire.

\section{MRI assessment and measurement}

The most lateral image of the sagittal MRI demonstrating a complete Y-shaped view of the scapula was utilized to evaluate the appearance of the SSP muscle belly. ${ }^{6,15}$ Selected digital images were then assessed using a SYNAPSE medical imaging system (Fujifilm Medical Co, Stamford, CT, USA) by two fellowship trained shoulder surgeons. The MRI measurements included the "occupation ratio", the "tangent sign", and the "location index" created for the current study (see below). Pre and postoperative results were compared and correlated with tear patterns.

The occupation ratio was defined as the ratio of the surface of the SSP muscle to the surface of the entire SSP fossa. ${ }^{6}$ The surface was measured by outlining the circumference of the muscle and fossa. The tangent sign was used with a straight reference line drawn to connect the superior tips of the scapula spine and the coracoid process and divided into three grades (1: negative, 2: borderline, 3: positive). ${ }^{7,14}$

The SSP muscle location (ie, the location index) was defined as the location of the muscle belly in anterior-posterior $(\mathrm{A}-\mathrm{P})$ direction relative to the center of the reference line (ie, the same line used to define the tangent sign). This was performed by drawing a line parallel to the reference line representing the maximum A-P width of muscle belly (Figure 1). The A-P distance of the posterior portion (posterior to the middle of the reference line) of the muscle was divided by the A-P width of the entire muscle belly (ie, the index is $>0.5$ if the center of the muscle is located posterior to the middle in the fossa and $<0.5$ when located anteriorly). Our preliminary SSP location index assessment on 50 shoulders (16-78 years old, 27 males and 23 females) without a rotator cuff tear revealed a mean index of $0.500 \pm 0.019$, indicating the

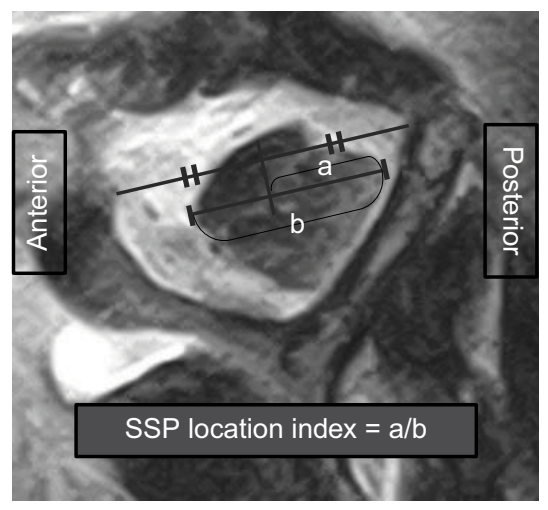

Figure I Measurement of SSP location index.

Note: The A-P distance of the posterior portion (a) of the muscle was divided by the A-P width of the entire muscle (b).

Abbreviations: SSP, supraspinatus; A-P, anterior-posterior.

index is $\sim 0.5$ if there is no significant atrophy or retraction of the SSP muscle. This group was utilized later as control for comparison with the study group.

In order to assess the reproducibility of the location index, preoperative MRIs of all the study cases (ie, 59 tears) were assessed twice by the first author $>6$ months apart, and once by a co-author, both of whom were blinded to the intraoperative details of each case. Intra-class correlation coefficient (ICC) analysis of preoperative location index revealed excellent agreement in both intra and interobserver reliability; $\operatorname{ICC}(1,1)=0.96(0.93,0.98), \operatorname{ICC}(2,1)=0.99$ $(0.98,0.99){ }^{18}$

\section{Operative record review and tear pattern assessment}

Operative records and intraoperative arthroscopic pictures were reviewed to obtain information of the tendons involved, repairability, tear patterns, and additional concomitant procedures performed (eg, biceps tenotomy, footprint medialization, teres minor advancement). Tear patterns were classified into 1) crescent-shaped, 2) L-shaped, 3) reverse L-shaped, and 4) U-shaped tears, as described in the literature. ${ }^{1,8}$ In this study, when the direction of reduction was mainly from medial to lateral, tears were defined as "crescent-shaped" or "U-shaped" tears. Tears were classified as "L-shaped" if the tendon was reduced from posteromedial to anterolateral direction with the use of an anterior apex anchor inserted at the anterolateral corner of the greater tuberosity. In contrast, "reverse L-shaped" tears were defined as tears with the tendon reduced from anteromedial to posterolateral direction with the use of a posterior apex anchor inserted at the posterolateral corner of the footprint. 


\section{Statistical analysis}

Results are shown as mean with $\mathrm{SD}$, range, or 95\% CI depending on the type of data. A paired $t$-test was used to determine significant differences between preoperative and postoperative measures. Welch's $t$-test was used to determine a significant difference of the SSP location index between reverse L-shaped tears and other tear patterns, as well as between the study and control groups. ANOVA followed by post hoc Tukey's test was utilized for univariate multigroup comparison among different tear patterns. Statistical significance was set at $P<0.05$. Receiver operating characteristic (ROC) curve was analyzed to calculate the area under the curve (AUC) and the cutoff value of the SSP location index where the sum of specificity and sensitivity was maximized. ICC analyses were also performed for the location index as previously explained. All statistical analyses were performed in consultation with a co-author who has experience in medical statistics using the combination of SPSS Statistics software, version 24 (IBM Corporation, Armonk, NY, USA) and EZR (Saitama Medical Center, Jichi Medical University, Saitama, Japan), which is a graphical user interface for $\mathrm{R}$ (The R Foundation for Statistical Computing, Vienna, Austria). ${ }^{19}$

\section{Results}

\section{Patient demographics}

Patient demographics are summarized in Table 1. Among the 158 arthroscopic rotator cuff repairs performed during the study period, 59 shoulders (58 patients, 20 females and 38 males, mean age 65.0 years) demonstrated large to massive tears preoperatively on MRI. The mean tear size was $3.7 \mathrm{~cm}$. Intraoperatively, the tendons involved were: SSP in nine shoulders, SSP and ISP in 26 shoulders, subscapularis

Table I Patient demographics

\begin{tabular}{|l|l|}
\hline Number of cases & $\begin{array}{l}59 \text { tears } \\
\text { (58 patients) }\end{array}$ \\
\hline Age (years) & $65.0(4 \mathrm{I}-78)$ \\
\hline Gender (M/F) & $20 / 38$ \\
\hline Tear size (cm) & $3.7(3.0-6.0)$ \\
\hline Tendons involved & SSP only: 9 \\
& SSc + SSP: II \\
& SSP + ISP: 26 \\
& SSc + SSP + ISP: I3 \\
\hline Tear patterns & Crescent: 7 \\
& L: 6 \\
& Reverse L: 5 \\
& U: 4I \\
\hline
\end{tabular}

Abbreviations: SSc, subscapularis; SSP, supraspinatus; ISP, infraspinatus; M/F, male/ female.
(SSc) and SSP in 11 shoulders, and SSc, SSP, and ISP in 13 shoulders. The tear pattern was deemed crescent-shaped in seven shoulders, U-shaped in 41 shoulders, L-shaped in six shoulders, and reverse L-shaped in five shoulders. Tears were reparable in 51 shoulders and irreparable in 8 shoulders. Footprint medialization $(5-10 \mathrm{~mm})$ on the greater tuberosity was performed in seven of the 51 reparable tears due to excessive tension on the repair. Interval slides in continuity were added to mobilize the torn tendons in 25 of 59 shoulders. While the mean tear size was larger in the tears which required interval slides than the tears which did not (3.6 and $3.3 \mathrm{~cm}$, respectively, $P=0.034$ ), the presence of interval slide was not associated with the tear pattern or the location index.

\section{SSP occupation ratio and tangent sign}

The SSP occupation ratio increased from $0.42 \pm 0.12$ preoperatively to $0.48 \pm 0.10$ postoperatively $(P<0.0001)$ (Table 2 ). The tangent sign was borderline or positive in $86.4 \%$ of patients ( 51 of 59 tears) preoperatively, and in $15.3 \%$ (9 of 59 tears) of patients postoperatively. Of the eight irreparable tears, the tangent sign was positive in seven tears and borderline in one tear. The grade of tangent sign improved in $71.2 \%$ (42 of 59 tears) after surgery, and none became worse (Table 2).

\section{SSP location index}

Overall, the center of the SSP muscle was located posteriorly with a location index of $0.647 \pm 0.125$ preoperatively, which was significantly greater than the control shoulders (ie, without a rotator cuff tear) $(0.500 \pm 0.019, P<0.0001)$. After repair, the SSP muscle shifted anteriorly with an index of $0.549 \pm 0.081(P<0.0001)$, which was still slightly posterior but closer to the middle of the fossa in A-P direction (Table 2). However, when divided by tear pattern, reverse L-shaped tears preoperatively showed a significantly lower index $(0.512 \pm 0.045)$, closer to the middle of the fossa, than the other three tear patterns $(0.660 \pm 0.122, P=0.00017)$ or U-shaped tears $(0.669 \pm 0.125, P=0.031)$ (Figure $2 \mathrm{~A})$. The location index increased to $0.552 \pm 0.063$ postoperatively, with no significant differences among tear patterns (Figure $2 \mathrm{~B}$ ). This resulted in an exceptional posterior shift in

Table 2 Perioperative changes of supraspinatus muscle appearance (results shown as mean \pm SD)

\begin{tabular}{|l|l|l|l|}
\hline $\begin{array}{l}\text { Measures on } \\
\text { sagittal MRI }\end{array}$ & Preoperative & Postoperative & $P$-value \\
\hline Occupation ratio & $0.42 \pm 0.12$ & $0.48 \pm 0.10$ & $<0.000 \mathrm{I}$ \\
\hline Tangent sign $(+\sim \pm)$ & $86.4 \%(5 \mathrm{I} / 59)$ & $\mathrm{I} 5.3 \%(9 / 59)$ & - \\
\hline Location index & $0.65 \pm 0.12$ & $0.55 \pm 0.08$ & $<0.000 \mathrm{I}$ \\
\hline
\end{tabular}



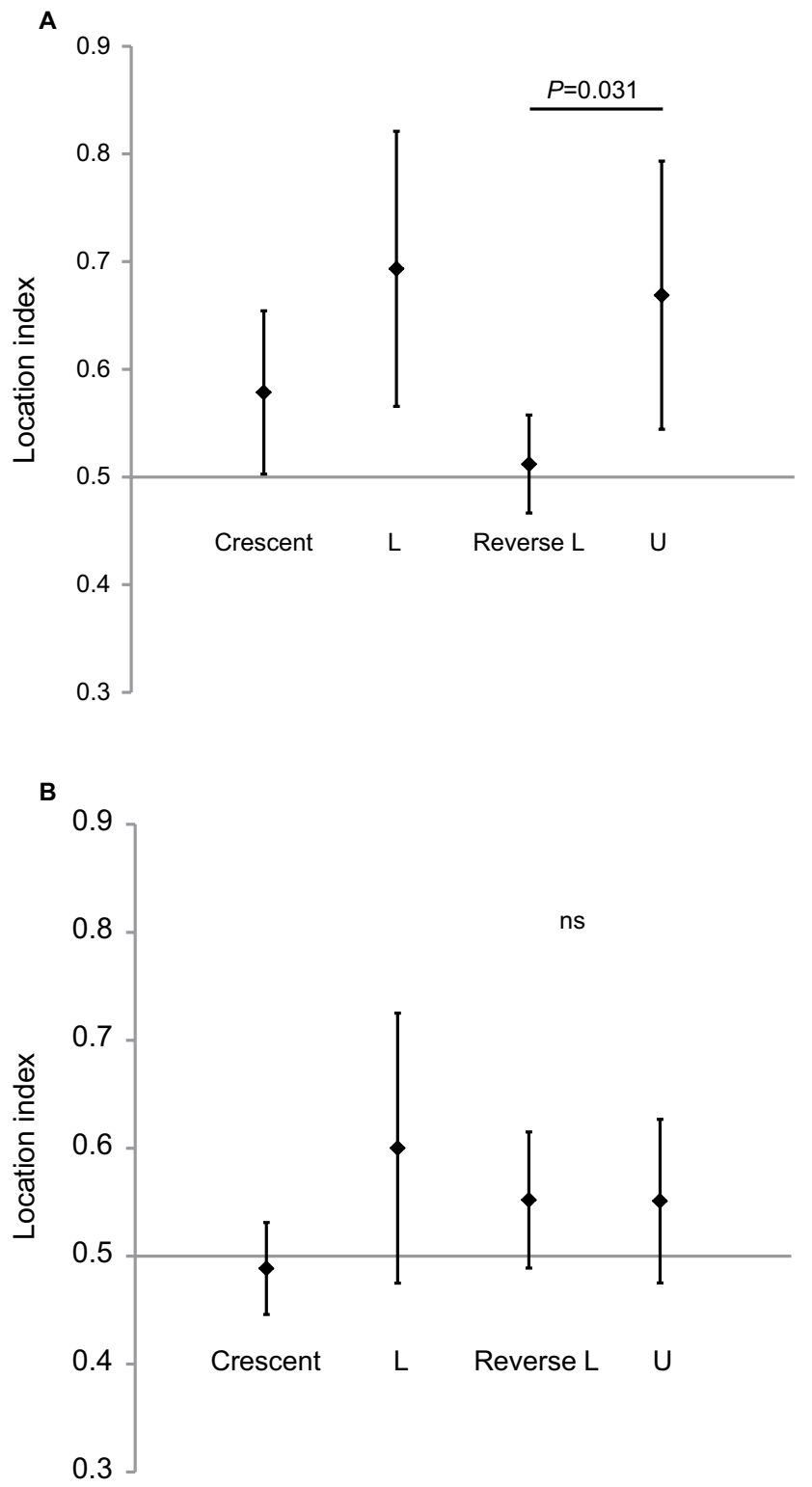

Figure 2 Preoperative (A) and postoperative (B) SSP location index by tear patterns (results shown as mean $\pm 95 \% \mathrm{Cl}$ ).

Abbreviations: SSP, supraspinatus; ns, not significant.

reverse L-shaped tears as opposed to the other tears, where the changes in the location index were $0.040 \pm 0.023$ and $-0.111 \pm 0.099$, respectively $(P<0.0001)$ (Figure 3$)$.

The ROC curve of the location index revealed a cutoff value of 0.56 (AUC 0.899 , specificity 0.796 , sensitivity 1.000) for the highest overall accuracy of 0.814 to distinguish a reverse L-shaped tear from other tear patterns (Figure 4).

\section{Discussion}

The most important finding of this study was that the SSP muscle belly was generally located more posteriorly in large

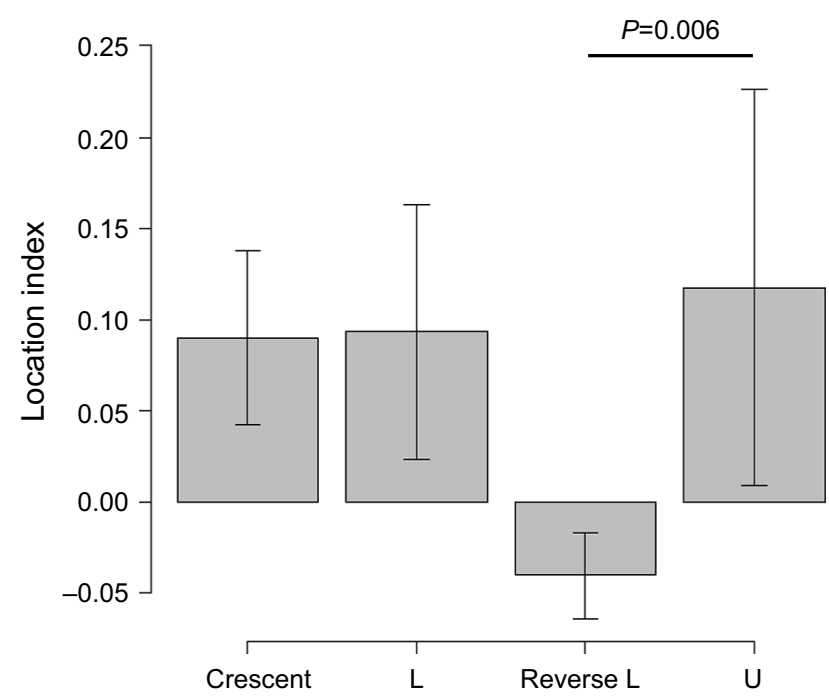

Figure 3 Perioperative changes in SSP location index by tear patterns (results shown as mean $\pm S D$ ).

Abbreviation: SSP, supraspinatus.

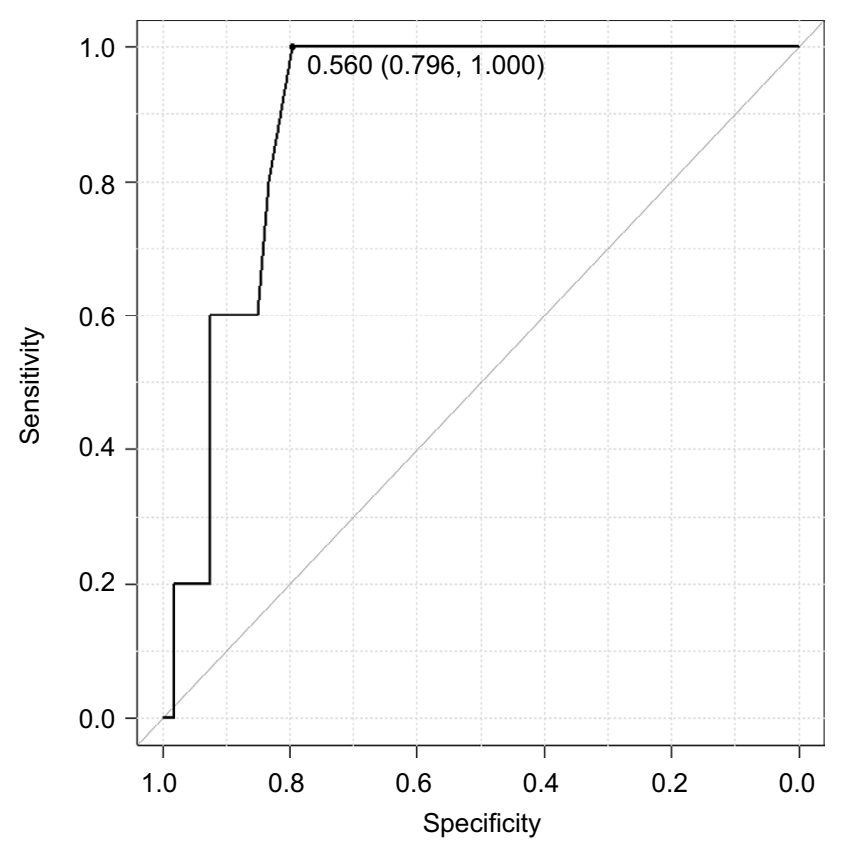

Figure 4 ROC curve analysis of SSP location index to predict reverse L-shaped tears or other tear patterns (results shown as mean $\pm S D$ ).

Abbreviations: SSP, supraspinatus; ROC, receiver operating characteristic.

to massive rotator cuff tears than in the shoulders without a rotator cuff tear. This suggests that tearing of the rotator cuff typically results in pulling of the SSP muscle-tendon unit posteriorly as the tear becomes larger and retracts. This posterior shift of the SSP muscle belly may be the result of the SSP muscle being pulled by the ISP muscle in a posterior direction. ${ }^{20,21}$ 
Interestingly, arthroscopic repair of these retracted tears normalized the appearance of the muscle belly immediately postoperatively. These results suggest that there is an improvement in the SSP muscle position relative to the SSP fossa in all three dimensions. Although the occupation ratio was originally described to measure muscle atrophy, more recently it has been suggested that it may also be utilized to describe muscle retraction or restoration, which involves the perioperative reduction of the muscle in a medial to lateral direction. ${ }^{6,14}$ Similarly, the tangent sign is another method, which also describes muscle atrophy and/or retraction, although this method strictly measures the atrophy/retraction in a vertical direction.

We described the location index which measures the anterior to posterior position of the SSP muscle. The location index is altered by the perioperative shift of rotator cuff muscle in the anterior to posterior direction during repair. In this study, the normalization in all three measures suggests that repairing the cuff tendon restored the SSP muscle back to a more anatomic location relative to the SSP fossa immediately after repair. Although restoring the muscle location to a more anatomic position seems logical, because of the lack of clinical evaluation (in this imaging study) it is unclear whether these results potentially improve function.

While preoperative assessment of the cuff muscles correlates with clinical outcomes and the repairability of a tear, ${ }^{5,12,13}$ to our knowledge, no studies have focused on the muscle location in the fossa or its perioperative changes. As hypothesized, this study demonstrated that rotator cuff repair resulted in relocating the SSP muscle belly closer to the center of the fossa, despite its variable preoperative position. Potentially preoperatively identifying the location of the SSP muscle belly within the fossa may be clinically predictive of the direction of reduction, although the final tear pattern and reduction should be confirmed intraoperatively.

Interestingly, in reverse L-shaped tears the SSP muscle was located more anteriorly before surgery, and shifted posteriorly after surgery, as opposed to the other tear patterns (ie, crescent-shaped, L-shaped, U-shaped tears), which demonstrated a common anterior shift. Reverse L-shaped tears are relatively rare ${ }^{10}$ and in our study represented $8.3 \%$ of large to massive tears. Because of their relative paucity, reverse L-shaped tears can be technically challenging since the tendon requires an anteromedial to posterolateral reduction while the typical direction of reduction in other tear patterns is from posteromedial to anterolateral. Inappropriate tear pattern recognition may place undue tension on the repair or may inappropriately deem a tear irreparable..$^{22,23}$ Potentially preoperatively identifying the location of the SSP muscle belly within the fossa may be clinically predictive of the tear pattern particularly for posterior L-shaped tears. While the final tear pattern should be confirmed intraoperatively, the current results may provide preoperative suspicion of the eventual tear pattern.

The presented SSP location index is a simple and reliable measure to quantify the location and shift of the SSP muscle. The results of our ROC curve analysis imply that, in retracted SSP tears, the tear would unlikely be a reverse L-shaped tear if the location index is over 0.56 (ie, if the muscle is located posteriorly), whereas there is a high chance of encountering a reverse L-shaped tear if the index is under 0.56 (ie, if the muscle is located close to the center or even anteriorly). Thus, the SSP location index may help predict the tear pattern preoperatively, and therefore assist in the planned reduction intraoperatively. Further studies, however, are required to validate this measure in a prospective fashion.

There are several limitations to this study due to the nature of the retrospective imaging study design, along with multiple factors involving the outcomes of this group of patients. First, it is unclear whether the recovery of the muscle location shown immediately postoperatively is maintained over time. While recovery in muscle degeneration over time after repair has been reported, ${ }^{15}$ the location of the muscle belly was not investigated in this study. Furthermore, the focus of this study was only on the SSP muscle belly in one sagittal oblique image. Therefore, study of the other rotator cuff muscles (ie, SSc, ISP, teres minor) or other sequential slices of the SSP may also provide valuable information. Second, while the total number of tears evaluated in the study was 59 , the number of reverse L-shaped tears was relatively small $(8.5 \%)$ and may not be a representative of the shifting patterns of the muscle belly. However, even with the small number of cases, statistical significance was obtained due to the consistent shifting pattern observed in reverse L-shaped tears. Third, neither postoperative clinical function nor tendon healing was determined. Therefore, it is unclear how restoration of the location of the SSP muscle affects these outcomes.

\section{Conclusion}

The position of the SSP muscle belly changes with large to massive rotator cuff tearing and following rotator cuff repair. Reverse L-shaped tears demonstrated a particular pattern of preoperative location and perioperative shift, when compared to the other tear patterns. The location of the SSP muscle belly may predict tear patterns of large to massive rotator cuff tears. 


\section{Acknowledgment}

The authors acknowledge research assistant Ms Yukiko Tago and Ms Wakako Morimoto for assisting with patient followup and data collection.

\section{Author contributions}

YO contributed to the conception and design of the study; data acquisition, analysis, and interpretation; and drafting and revising the work critically for important intellectual content. TM contributed to data acquisition, analysis, and interpretation; and drafting and revising the manuscript. SY contributed to data analysis and interpretation, and revising the manuscript. KKWT, NK, and AK contributed to the data interpretation, and revising the manuscript. IKYL contributed to the study design, data interpretation, and drafting and revising the manuscript. All authors contributed to data analysis, drafting and revising the article, gave final approval of the version to be published, and agree to be accountable for all aspects of the work.

\section{Disclosure}

YO has received a manuscript fee from Smith \& Nephew for work outside the submitted study. The other authors report no conflicts of interest in this work.

\section{References}

1. Burkhart SS, Lo IK, Ik L. Arthroscopic rotator cuff repair. J Am Acad Orthop Surg. 2006;14(6):333-346.

2. Cofield RH. Subscapular muscle transposition for repair of chronic rotator cuff tears. Surg Gynecol Obstet. 1982;154(5):667-672.

3. Patte D. Classification of rotator cuff lesions. Clin Orthop Relat Res. 1990;254:81-86.

4. Fuchs B, Weishaupt D, Zanetti M, Hodler J, Gerber C. Fatty degeneration of the muscles of the rotator cuff: assessment by computed tomography versus magnetic resonance imaging. J Shoulder Elbow Surg. 1999;8(6):599-605.

5. Goutallier D, Postel JM, Bernageau J, Lavau L, Voisin MC. Fatty muscle degeneration in cuff ruptures. Pre- and postoperative evaluation by CT scan. Clin Orthop Relat Res. 1994;304:78-83.

6. Thomazeau H, Rolland Y, Lucas C, Duval JM, Langlais F. Atrophy of the supraspinatus belly. Assessment by MRI in 55 patients with rotator cuff pathology. Acta Orthop Scand. 1996;67(3):264-268.

7. Zanetti M, Gerber C, Hodler J. Quantitative assessment of the muscles of the rotator cuff with magnetic resonance imaging. Invest Radiol. 1998;33(3):163-170.
8. Nho SJ, Ghodadra N, Provencher MT, Reiff S, Romeo AA. Anatomic reduction and next-generation fixation constructs for arthroscopic repair of crescent, L-shaped, and U-shaped rotator cuff tears. Arthroscopy. 2009;25(5):553-559.

9. Park JY, Jung SW, Jeon SH, Cho HW, Choi JH, Oh KS. Arthroscopic repair of large U-shaped rotator cuff tears without margin convergence versus repair of crescent- or L-shaped tears. Am J Sports Med. 2014;42(1):103-111.

10. Sallay PI, Hunker PJ, Lim JK. Frequency of various tear patterns in fullthickness tears of the rotator cuff. Arthroscopy. 2007;23(10):1052-1059.

11. Ellman H. Arthroscopic Shoulder Surgery and Related Disorders. Philadelphia: Lea \& Febiger; 1993.

12. Thomazeau H, Boukobza E, Morcet N, Chaperon J, Langlais F. Prediction of rotator cuff repair results by magnetic resonance imaging. Clin Orthop Relat Res. 1997;344:275-283.

13. Warner JJ, Higgins L, Parsons IM, Dowdy P. Diagnosis and treatment of anterosuperior rotator cuff tears. J Shoulder Elbow Surg. 2001;10(1):37-46.

14. Jo CH, Shin JS. Changes in appearance of fatty infiltration and muscle atrophy of rotator cuff muscles on magnetic resonance imaging after rotator cuff repair: establishing new time-zero traits. Arthroscopy 2013;29(3):449-458.

15. Hamano N, Yamamoto A, Shitara H, et al. Does successful rotator cuff repair improve muscle atrophy and fatty infiltration of the rotator cuff? A retrospective magnetic resonance imaging study performed shortly after surgery as a reference. J Shoulder Elbow Surg. 2017;26(6): 967-974.

16. Hamada K, Fukuda H, Mikasa M, Kobayashi Y. Roentgenographic findings in massive rotator cuff tears. A long-term observation. Clin Orthop Relat Res. 1990;254:92-96.

17. Lo IK, Burkhart SS. The interval slide in continuity: a method of mobilizing the anterosuperior rotator cuff without disrupting the tear margins. Arthroscopy. 2004;20(4):435-441

18. Clopton N, Dutton J, Featherston T, Grigsby A, Mobley J, Melvin J. Interrater and intrarater reliability of the modified Ashworth scale in children with hypertonia. Pediatr Phys Ther. 2005;17(4):268-274.

19. Kanda Y. Investigation of the freely available easy-to-use software 'EZR' for medical statistics. Bone Marrow Transplant. 2013;48(3): $452-458$.

20. Kim HM, Dahiya N, Teefey SA, et al. Location and initiation of degenerative rotator cuff tears: an analysis of three hundred and sixty shoulders. J Bone Joint Surg Am. 2010;92(5):1088-1096.

21. Mochizuki T, Sugaya H, Uomizu M, et al. Humeral insertion of the supraspinatus and infraspinatus. new anatomical findings regarding the footprint of the rotator cuff. Surgical technique. J Bone Joint Surg Am. 2009;91(Suppl 2):1-7.

22. Burkhart SS, Hartzler RU. Arthroscopic rotator cuff repair: how to avoid Retear. Arthroscopy. 2019;35(1):12-13.

23. Pastor MF, Kraemer M, Schwarze M, Hurschler C, Smith T, Wellmann $\mathrm{M}$. The effect of rotator cuff malreduction on tendon tension: an evaluation of a custom-made digital tensiometer clamp. Arch Orthop Trauma Surg. 2018;138(2):219-225.
Open Access Journal of Sports Medicine

\section{Publish your work in this journal}

The Open Access Journal of Sports Medicine is an international, peer-reviewed, open access journal publishing original research, reports, reviews and commentaries on all areas of sports medicine. The journal is included on PubMed. The manuscript management system is completely online and includes a very quick and fair

\section{Dovepress}

peer-review system. Visit http://www.dovepress.com/testimonials.php to read real quotes from published authors. 